\title{
PARTIAL MANIFESTATION OF A GENE IN COMPLETE THREE AND HIGHER POINT BACKCROSS DATA
}

\author{
P. A. PARSONS \\ Deportment of Genetics, Combridge University
}

Received 20.iv.56

\section{INTRODUCTION}

IMPERFECT penetrance of a gene in a linkage experiment leads to considerable disturbance in the data, frequently of far greater magnitude than that caused by differential viability, which usually needs very large numbers to reveal its presence. The problem of estimating the degree of misclassification was considered by Smith (1937), but of far greater importance is the question of finding accurate estimates of the recombination values. Two-point backcross data have been treated adequately by Bailey (1950) and Sánchez-Monge (1952) on theoretical grounds. Fisher (1953a) used Bailey's method to estimate the recombination fraction between polydactyly $(p y)$, which is imperfectly penetrant, and leaden $(l n)$ in the thirteenth chromosome of the house mouse, but he modified Bailey's estimates of the sampling variances.

In this paper it is proposed to extend the methods developed for two-point data to complete three and higher point backcross data. By complete we shall mean a backcross in which data have been collected for all possible heterozygous parents. In the case of a three-point test there are four possible heterozygotes, and in a fourpoint there are eight.

Furthermore, this paper considers the simplest possible situation, which is that of the partial manifestation of one gene unaffected by the partial manifestation or differential viability of any other gene. Differential viability in a linkage experiment is often largely catered for without further calculation if the experiment is adequately balanced (i.e. there are approximately an equal number of zygotes in each of the possible mating types).

Before considering three and higher point data, the method proposed by Fisher (1953a) for two-point data will be reviewed, as the treatment of higher point data merely extends this.

\section{TWO-POINT DATA}

Consider a backcross $\frac{A B}{a b}=\frac{a b}{a b}$ in which $a$ is misclassified such that $a \rightarrow A$, the wild type allele. Let $\lambda$ be the misclassification and $p$ the recombination fraction between $A$ and $B$ such that $p+q=\mathrm{I}$.

Observations and expectations may now be drawn up :-

$\begin{array}{cccccc} & A B & a b & A b & a B & \text { Total } \\ \text { Observed } & a & b & c & d & n \\ \text { Expected } & \frac{1}{2}(q+\lambda p) & \frac{1}{2} q(\mathrm{I}-\lambda) & \frac{1}{2}(p+\lambda q) & \frac{1}{2} p(\mathrm{I}--\lambda) & \mathbf{I}\end{array}$


To find the estimates $\hat{p}$ and $\hat{\lambda}$ we may proceed as follows :$p(\mathrm{I}-\lambda)=\frac{d}{a+d}=a=$ proportion of $a$ in $B$ phenotypes $q(\mathrm{I}-\lambda)=\frac{b}{b+c}=\beta=$ proportion of $a$ in $b$ phenotypes.

Adding

$$
\mathrm{I}-\hat{\lambda}=\alpha+\beta
$$

and

$$
\hat{p}=\frac{a}{a+\beta}
$$

these forms being equivalent to those given by Bailey (1950).

The quantities $a$ and $\beta$ are respectively proportions observed of the manifesting class out of the number $a+d$ of $B$ phenotypes, and the number $b+c$ of $b$ phenotypes. Thus $a$ and $\beta$ can be regarded as determined conditional on $a+d$ and $b+c$ respectively, and so have binomial variances.

$$
V(\alpha)=\frac{\alpha(\mathrm{I}-\alpha)}{a+d}, V(\beta)=\frac{\beta(\mathrm{I}-\beta)}{b+c}
$$

The variances $V(p)$ and $V(\lambda)$ are calculated as follows.

From equation (2.I)

$$
V(\lambda)=V(\mathrm{I}-\lambda)=V(\alpha+\beta)
$$

Since $\alpha$ and $\beta$ are confined to two non-overlapping totals, the covariance term in $V(\alpha+\beta)$ will be zero. Therefore

$$
V(\lambda)=V(\alpha)+V(\beta)
$$

We may find $V(p)$ using the relationship :-

$$
V(p)=\left(\frac{\partial p}{\partial \alpha}\right)^{2} V(\alpha)+\left(\frac{\partial p}{\partial \beta}\right)^{2} V(\beta)+2 \frac{\partial p}{\partial \alpha} \cdot \frac{\partial p}{\partial \beta} V(\alpha \beta)
$$

(Fisher, 1953b)

As $\alpha$ and $\beta$ are independent, the covariance term in this expression is zero and we obtain :-

$$
V(p)=\frac{\beta^{2} V(\alpha)+\alpha^{2} V(\beta)}{(\alpha+\beta)^{4}}
$$

The variances $V(p)$ and $V(\lambda)$ are calculated in terms of $\alpha, \beta$, $V(\alpha)$ and $V(\beta)$ which are in turn dependent upon the observed numbers $a, b, c, d$. Hence $V(p)$ and $V(\lambda)$ are calculated from the information realised. In the formulæ $(2.7,2.8)$ given by Bailey (1950) $V(p)$ and $V(\lambda)$ are based upon the information expected (for a given total $n$ ).

Bailey gives :-

$$
\begin{aligned}
& V(\lambda)=\frac{2(\mathrm{I}-\lambda)}{n}\{2 p q+\lambda(\mathrm{I}-2 p q)\} \\
& V(p)=\frac{2 p q}{n}\left\{\frac{\mathrm{I}}{\mathrm{I}-\lambda}-2 p q\right\}
\end{aligned}
$$

which are equivalent to Fisher's formulx $(2.5,2.6)$ when

$$
a+d=b+c=\frac{1}{2} n
$$


This relation (2.9) is derived from the expectations of the four phenotypes $A B, a b, A b$ and $a B$.

Hence $V(p)$ and $V(\lambda)$ calculated as information realised will almost certainly be different to the information expected and in fact are only equivalent when the condition (2.9) holds. Fisher (1953a) gives a case for the linkage between $p y$ which is imperfectly penetrant and $l n$ for males in coupling where this condition holds. In this set of data the goodness of fit $\chi^{2}{ }_{1}$ is zero.

TABLE

Expectations, admitting misclassification of gene a

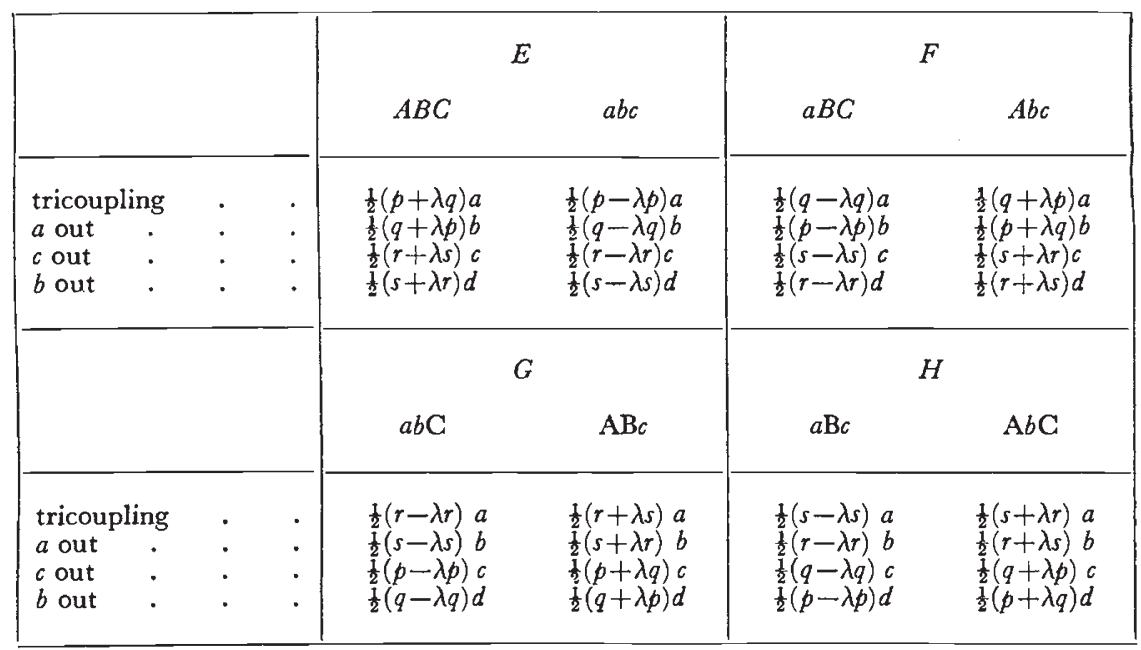

\section{THREE-POINT DATA}

A three-point backcross will now be considered in which the four possible triply heterozygous parents are represented. Let the two segments between the three linked genes $a, b, c$ be (I) and (2). Let $p, q, r, s$ be the frequencies of the modes of gamete formation (o), (1), (2), (12) and $a, b, c, d$ be proportional to the number of zygotes in each mating type such that

$$
\begin{aligned}
& p+q+r+s=\mathrm{I} \\
& a+b+c+d=\mathrm{I}
\end{aligned}
$$

and let $\lambda=$ misclassification of gene $a$ such that $a \rightarrow A$.

Let $E, F, G$ and $H$ represent the four pairs of complementary genotypes :-

$$
\begin{aligned}
& \text { E } A B C, a b c \\
& F \quad a B C, A b c \\
& G \quad a b C, A B c \\
& H a B c, A b C
\end{aligned}
$$

For each complementary pair, admitting misclassification of gene $a$, expectations may be drawn up (table I). 
These expectations may be re-arranged as a $4 \times 4$ Latin Square of the form :-

$$
\begin{array}{llll}
E & F & G & H \\
F & E & H & G \\
G & H & E & F \\
H & G & F & E
\end{array}
$$

in which the columns correspond to the four modes of gamete formation, the rows correspond to the four mating types, and the letters to the four pairs of complementary genotypes. The re-arranged expectations, after adding the members of each complementary pair, are tabulated below (table 2).

TABLE 2

Expectations arranged in a Latin Square

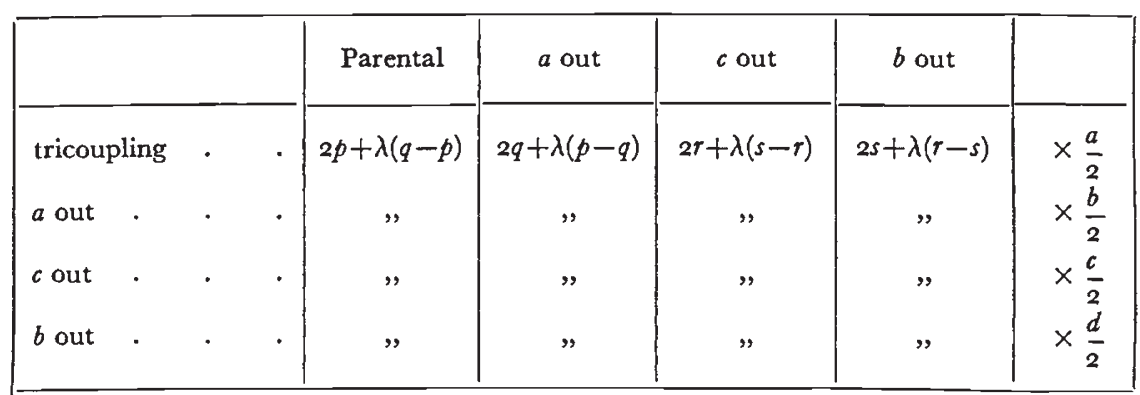

The expected column totals in table 2 may be equated to the observed column totals which we can represent by $P, Q, R$ and $S$ respectively. We obtain (remembering that $a+b+c+d=\mathrm{I}$ ) four equations :-

Adding (3.I) and (3.2)

$$
\begin{aligned}
& p+\frac{1}{2} \lambda(q-p)=P . \quad . \quad . \quad \text {. (3.1) } \\
& q+\frac{1}{2} \lambda(p-q)=Q \text {. . . . . (3.2) } \\
& r+\frac{1}{2} \lambda(s-r)=R \text {. . . . . (3.3) } \\
& s+\frac{1}{2} \lambda(r-s)=S \text {. . . . . (3.4) }
\end{aligned}
$$

and substituting in $(3.1)$

$$
p+q=P+Q
$$

$$
\begin{aligned}
& p=\frac{P-\frac{1}{2} \lambda(P+Q)}{\mathrm{I}-\lambda} \\
& \text { and } \\
& q=\frac{Q-\frac{1}{2} \lambda(P+Q)}{\mathrm{I}-\lambda} \\
& \left.r=\frac{R-\frac{1}{2} \lambda(R+S)}{\mathrm{I}-\lambda}\right\} \\
& s=\frac{S-\frac{1}{2} \lambda(R+S)}{\mathrm{I}-\lambda}
\end{aligned}
$$


From the calculated values of $p, q, r$ and $s$ (3.5), which are the four modes of gamete formation, the three possible recombination values $(q+r),(r+s)$, and $(q+s)$ may be calculated by simple addition since $p+q+r+s=\mathbf{I}$.

The misclassification, $\lambda$, may be calculated by a method analogous to that employed for two-point data.

Let

$$
\begin{aligned}
a p+b q+c r+d s & =\mathcal{J} \\
a q+b p+c s+d r & =K \\
a r+b s+c p+d q & =L \\
a s+b r+c q+d p & =M
\end{aligned}
$$

From table I

$$
\begin{aligned}
& a \text { in } B C=\frac{\text { total observed } a B C}{\text { total observed }(a B C+A B C)}=\frac{K(\mathrm{I}-\lambda)}{\mathcal{J}+K}=a \\
& a \text { in } b c=\frac{a b c}{a b c+A b c}=\frac{\mathcal{F}(\mathrm{I}-\lambda)}{\mathcal{J}+K}=\beta \\
& a \text { in } b C=\quad \frac{a b C}{a b C+A b C}=\frac{L(\mathrm{I}-\lambda)}{L+M}=\gamma \\
& a \text { in } B c=\frac{a B c}{a B c+A B c}=\frac{M(\mathrm{I}-\lambda)}{L+M}=\delta
\end{aligned}
$$

Adding

and also

$$
\mathrm{I}-\lambda=\frac{1}{2}(\alpha+\beta+\gamma+\delta) \quad . \quad .
$$

$$
V(\mathrm{I}-\lambda)=\frac{1}{4}[V(\alpha)+V(\beta)+V(\gamma)+V(\delta)] .
$$

where $V(\alpha), V(\beta), V(\gamma)$ and $V(\delta)$ are calculated as for the two-point situation.

The variances of the recombination fractions $(r+s),(q+r)$, and $(q+s)$ are most easily found by considering them separately as twopoint data.

The recombination fraction $(r+s)$ measures recombination in segment $b c$ and is therefore independent of the partial manifestation. of gene $a$. The variance is therefore found in the normal way :-

$$
V(r+s)=\frac{(r+s)(\mathrm{I}-\overline{r+s})}{n}
$$

Now $(q+s)$ measures recombination in segment $a b$. In table $\mathrm{I}$, by ignoring gene $c$ we can calculate $\alpha_{1}$ and $\beta_{1}$ the proportion of $a$ in $B$ and $a$ in $b$ respectively. Thus we find :-

$$
V(q+s)=\frac{\beta_{1}^{2} V\left(a_{1}\right)+\alpha_{1}^{2} V\left(\beta_{1}\right)}{\left(a_{1}+\beta_{1}\right)^{4}}
$$

And similarly, by ignoring gene $b, V(q+r)$ may be found.

It must finally be noted that if gene $b$ or $c$ were misclassified instead of $a$, two similar but not identical sets of four expressions for $p, q$, $r$ and $s$ would be obtained. 


\section{FOUR-POINT DATA AND MORE}

The method for four-point data is merely an extension of that for three-point data. Let the three segments between the four linked genes $a, b, c, d$ be (1), (2) and (3). Let the frequencies of the eight modes of gamete formation (0), (1), (2), (3), (12), (13), (23) and (123) be $p, q, r, s, t, u, v$ and $w$ respectively such that

$$
p+q+r+s+t+u+v+w=\mathbf{I}
$$

By arranging the data from the eight possible four-fold heterozygotes in an $8 \times 8$ Latin Square eight equations can be derived. The following set of eight equations is derived for misclassification of gene $d$.

$$
\begin{aligned}
p+\frac{1}{2} \lambda(s-p) & =P \\
q+\frac{1}{2} \lambda(u-q) & =Q \\
r+\frac{1}{2} \lambda(v-r) & =R \\
s+\frac{1}{2} \lambda(p-s) & =S \\
t+\frac{1}{2} \lambda(w-t) & =T \\
u+\frac{1}{2} \lambda(q-u) & =U \\
v+\frac{1}{2} \lambda(r-v) & =V \\
w+\frac{1}{2} \lambda(t-w) & =W
\end{aligned}
$$

where $P, Q, R, S, T, U, V, W$ are the observed column totals of the $8 \times 8$ Latin Square.

From the above set of eight equations, the frequencies of the eight modes of gamete formation can be calculated. This method will be equally applicable to higher point data.

\section{SUMMARY}

A method for allowing for partial manifestation of a gene in complete three and higher point backcross data is proposed. This is needed in the treatment of four-point data such as that being collected in this Department for the chromosome XIII markers py, $\ln , f z$ (fuzzy) and $S p$ (Splotch) of the house mouse by Professor Sir Ronald Fisher.

Acknowledgment.-I wish to thank Professor Sir Ronald Fisher for many helpful discussions.

\section{REFERENCES}

BAILEY, N. T. J. 1950. The influence of partial manifestation on the detection of linkage. Heredity, 4, 327-336.

FISHER, R. A. 1953a. The linkage of polydactyly with leaden in the house mouse. Heredity, 7, $9 \mathrm{I}-95$.

FISHER, R. A. I953b. The Design of Experiments, 6th ed. Edinburgh : Oliver and Boyd.

SANGHEZ-MONGE, E. 1952. The estimation of linkage with incomplete penetrance. Heredity, 6, I 2 I-1 25.

SMITH, H. F. 1937. Test of significance for Mendelian ratios when classification is uncertain. Ann. Eug., 8, 94-95. 